\title{
Analysis of the inverse problem in a time fractional parabolic equation with mixed boundary conditions
}

\author{
Ebru Ozbilge ${ }^{1 *}$ and Ali Demir ${ }^{2}$
}

\author{
"Correspondence: \\ ebru.ozbilge@ieu.edu.tr \\ ${ }^{1}$ Department of Mathematics, \\ Faculty of Science and Literature, \\ Izmir University of Economics, \\ Sakarya Caddesi, No. 156, Balcova, \\ Izmir, 35330, Turkey \\ Full list of author information is \\ available at the end of the article
}

\begin{abstract}
This article deals with the mathematical analysis of the inverse coefficient problem of identifying the unknown coefficient $k(x)$ in the linear time fractional parabolic equation $D_{t}^{\alpha} u(x, t)=\left(k(x) u_{x}\right)_{x}, 0<\alpha \leq 1$, with mixed boundary conditions $u(0, t)=\psi_{0}(t), u_{x}(1, t)=\psi_{1}(t)$. By defining the input-output mappings $\Phi[\cdot]: \mathcal{K} \rightarrow C^{1}[0, T]$ and $\Psi[\cdot]: \mathcal{K} \rightarrow C[0, T]$, the inverse problem is reduced to the problem of their invertibility. Hence the main purpose of this study is to investigate the distinguishability of the input-output mappings $\Phi[\cdot]$ and $\Psi[\cdot]$. This work shows that the input-output mappings $\Phi[\cdot]$ and $\Psi[\cdot]$ have the distinguishability property. Moreover, the value $k(0)$ of the unknown diffusion coefficient $k(x)$ at $x=0$ can be determined explicitly by making use of measured output data (boundary observation) $k(0) u_{x}(0, t)=f(t)$, which brings greater restriction on the set of admissible coefficients. It is also shown that the measured output data $f(t)$ and $h(t)$ can be determined analytically by a series representation, which implies that the input-output mappings $\Phi[\cdot]: \mathcal{K} \rightarrow C^{1}[0, T]$ and $\Psi[\cdot]: \mathcal{K} \rightarrow C[0, T]$ can be described explicitly.
\end{abstract}

\section{Introduction}

The inverse problem of determining an unknown coefficient in a linear parabolic equation by using over-measured data has generated an increasing amount of interest from engineers and scientist during the last few decades. This kind of problem plays a crucial role in engineering, physics and applied mathematics. The problem of recovering an unknown coefficient or coefficients in the mathematical model of physical phenomena is frequently encountered. Intensive study has been carried out on this kind of problem, and various numerical methods have been developed in order to overcome the problem of determining an unknown coefficient or coefficients [1-9]. The inverse problem of unknown coefficients in a quasi-linear parabolic equations was studied by Demir and Ozbilge [5, 6]. Moreover, the identification of the unknown diffusion coefficient in a linear parabolic equation was studied by Demir and Hasanov [7].

Fractional differential equations are generalizations of ordinary and partial differential equations to an arbitrary fractional order. By linear time-fractional parabolic equation, we mean a certain parabolic-like partial differential equation governed by master equations containing fractional derivatives in time $[10,11]$. The research areas of fractional differential equations range from theoretical to applied aspects. The main goal of this study is

\section{Springer}

@2014 Ozbilge and Demir; licensee Springer. This is an Open Access article distributed under the terms of the Creative Commons Attribution License (http://creativecommons.org/licenses/by/2.0), which permits unrestricted use, distribution, and reproduction in any medium, provided the original work is properly cited. 
to investigate the inverse problem of determining an unknown coefficient $k(x)$ in a onedimensional time fractional parabolic equation. We first obtain the unique solution of this problem using the Fourier method of separation of variables with respect to the eigenfunctions of the corresponding Sturm-Liouville eigenvalue problem under certain conditions [12]. As the next step, the noise-free measured output data are used to introduce the inputoutput mappings $\Phi[\cdot]: \mathcal{K} \rightarrow C^{1}[0, T]$ and $\Psi[\cdot]: \mathcal{K} \rightarrow C[0, T]$. Finally, we investigate the distinguishability of the unknown coefficient via the above input-output mappings $\Phi[\cdot]$ and $\Psi[\cdot]$.

Consider now the following initial boundary value problem:

$$
\left\{\begin{array}{l}
D_{t}^{\alpha} u(x, t)=\left(k(x) u_{x}\right)_{x}, \quad 0<\alpha \leq 1,(x, t) \in \Omega_{T}, \\
u(x, 0)=g(x), \quad 0<x<1, \\
u(0, t)=\psi_{0}(t), \quad u_{x}(1, t)=\psi_{1}(t), \quad 0<t<T,
\end{array}\right.
$$

where $\Omega_{T}=\left\{(x, t) \in R^{2}: 0<x<1,0<t \leq T\right\}$ and the fractional derivative $D_{t}^{\alpha} u(x, t)$ is defined in the Caputo-Dzherbashyan sense $D_{t}^{\alpha} u(x, t)=\left(I^{1-\alpha} u^{\prime}\right)(t), 0<\alpha \leq 1, I^{\alpha}$ being the Riemann-Liouville fractional integral

$$
\left(I^{\alpha} f\right)(t)= \begin{cases}\frac{1}{\Gamma(\alpha)} \int_{0}^{t}(t-\tau)^{\alpha-1} f(\tau) d \tau, & 0<\alpha \leq 1, \\ f(t), & \alpha=0 .\end{cases}
$$

The left and right boundary value functions $\psi_{0}(t)$ and $\psi_{1}(t)$ belong to $C^{1}[0, T]$. The functions $0<c_{0} \leq k(x)<c_{1}$ and $g(x)$ satisfy the following conditions:

(C1) $k(x) \in C^{1}[0,1]$

(C2) $g(x) \in C^{2}[0,1], g(0)=\psi_{0}(0), g^{\prime}(1)=\psi_{1}(0)$.

Under these conditions, the initial boundary value problem (1) has the unique solution $u(x, t)$ defined in the domain $\bar{\Omega}_{T}=\left\{(x, t) \in R^{2}: 0 \leq x \leq 1,0 \leq t \leq T\right\}$ which belongs to the space $C\left(\bar{\Omega}_{T}\right) \cap W_{t}^{1}(0, T] \cap C_{x}^{2}(0,1)$. Moreover, it satisfies the equation, initial and boundary conditions. The space $W_{t}^{1}(0, T]$ contains the functions $f \in C^{1}(0, T]$ such that $f^{\prime}(t) \in L(0, T)$.

This kind of problem plays a crucial role in engineering, physics and applied mathematics since it is used successfully to model complex phenomena in various fields such as fluid mechanics, viscoelasticity, physics, chemistry and engineering. The problem of recovering an unknown coefficient or coefficients in the mathematical model of physical phenomena is frequently encountered.

Consider the inverse problem of determining the unknown coefficient $k(x)$ from the Neumann-type measured output data at the boundary $x=0$ :

$$
k(0) u_{x}(0, t)=f(t), \quad t \in(0, T]
$$

and the Dirichlet-type measured output data at the boundary $x=1$ :

$$
u(1, t)=h(t), \quad t \in(0, T] .
$$

Here $u=u(x, t)$ is the solution of parabolic problem (1). The functions $f(t)$ and $h(t)$ are assumed to be noise-free measured output data. In this context, parabolic problem (1) will 
be referred to as a direct (forward) problem with the inputs $g(x)$ and $k(x)$. It is assumed that the functions $f(t)$ and $h(t)$ belong to $C[0, T]$ and satisfy the consistency conditions $f(0)=k(0) g^{\prime}(0)$ and $g(1)=h(0)$.

By denoting $\mathcal{K}:=\left\{k(x) \in C^{1}[0,1]: c_{1}>k(x) \geq c_{0}>0, x \in[0,1]\right\} \subset C[0,1]$, the set of admissible coefficients $k(x)$, let us introduce the input-output mappings $\Phi[\cdot]: \mathcal{K} \rightarrow C^{1}[0, T]$ and $\Psi[\cdot]: \mathcal{K} \rightarrow C[0, T]$, where

$$
\Phi[k]=\left.k(x) u_{x}(x, t ; k)\right|_{x=0}, \quad k \in \mathcal{K}
$$

and

$$
\Psi[k]=\left.u(x, t ; k)\right|_{x=1}, \quad k \in \mathcal{K} .
$$

Then the inverse problem with the measured output data $f(t)$ and $h(t)$ can be formulated as follows:

$$
\Phi[k]=f, \quad f \in C^{1}(0, T], \quad \Psi[k]=h, \quad h \in C(0, T],
$$

which reduces the inverse problem of determining the unknown coefficient $k(x)$ to the problem of invertibility of the input-output mappings $\Phi[\cdot]$ and $\Psi[\cdot]$. Hence this leads us to investigate the distinguishability of the unknown coefficient via the above input-output mappings. We say that the mappings $\Phi[\cdot]: \mathcal{K} \rightarrow C^{1}[0, T]$ and $\Psi[\cdot]: \mathcal{K} \rightarrow C[0, T]$ have the distinguishability property if $\Phi\left[k_{1}\right] \neq \Phi\left[k_{2}\right]$ implies $k_{1}(x) \neq k_{2}(x)$ and the same holds for $\Psi[\cdot]$. This, in particular, means the injectivity of inverse mappings $\Phi^{-1}$ and $\Psi^{-1}$. In this paper, measured output data of Neumann type at the boundary $x=0$ and measured output data of Dirichlet type at the boundary $x=1$ are used in the identification of the unknown coefficient. In addition, in the determination of the unknown parameter, analytical results are obtained.

The paper is organized as follows. In Section 2, an analysis of the inverse problem with the single measured output data $f(t)$ at the boundary $x=0$ is given. An analysis of the inverse problem with the single measured output data $h(t)$ at the boundary $x=1$ is considered in Section 3. Finally, some concluding remarks are given in the last section.

\section{An analysis of the inverse problem with given measured data $f(t)$}

Consider now the inverse problem with one measured output data $f(t)$ at $x=0$. In order to formulate the solution of parabolic problem (1) by using the Fourier method of the separation of variables, let us first introduce an auxiliary function $v(x, t)$ as follows:

$$
v(x, t)=u(x, t)-\psi_{0}(t)-\psi_{1}(t) x, \quad x \in[0,1],
$$

by which we transform problem (1) into a problem with homogeneous boundary conditions. Hence the initial boundary value problem (1) can be rewritten in terms of $v(x, t)$ in the following form:

$$
\left\{\begin{array}{l}
D_{t}^{\alpha} v(x, t)-v_{x x}(x, t)=\left((k(x)-1) v_{x}(x, t)\right)_{x}-x D_{t}^{\alpha} \psi_{1}(t)-D_{t}^{\alpha} \psi_{0}(t)+k^{\prime}(x) \psi_{1}(t), \\
\quad(x, t) \in \Omega_{T}, \\
v(x, 0)=g(x)-\psi_{0}(0)-\psi_{1}(0) x, \quad 0<x<1, \\
v(0, t)=0, \quad v_{x}(1, t)=0, \quad 0<t<T .
\end{array}\right.
$$


The unique solution of the initial-boundary value problem can be represented in the following form [12]:

$$
\begin{aligned}
v(x, t)= & \sum_{n=1}^{\infty}\left\langle\zeta(\theta), \phi_{n}(\theta)\right| E_{\alpha, 1}\left(-\lambda_{n} t^{\alpha}\right) \phi_{n}(x) \\
& +\sum_{n=1}^{\infty}\left(\int_{0}^{t} s^{\alpha-1} E_{\alpha, \alpha}\left(-\lambda_{n} s^{\alpha}\right)\left\langle\xi(\theta, t-s), \phi_{n}(\theta)\right\rangle d s\right) \phi_{n}(x),
\end{aligned}
$$

where

$$
\begin{aligned}
& \zeta(x)=g(x)-\psi_{0}(0)-\psi_{1}(0) x, \\
& \xi(x, t)=\left((k(x)-1) v_{x}(x, t)\right)_{x}-x D_{t}^{\alpha} \psi_{1}(t)-D_{t}^{\alpha} \psi_{0}(t)+k^{\prime}(x) \psi_{1}(t) .
\end{aligned}
$$

Moreover, $\left\langle\zeta(\theta), \phi_{n}(\theta)\right\rangle=\int_{0}^{1} \phi_{n}(\theta) \zeta(\theta) d \theta, E_{\alpha, \beta}$ being the generalized Mittag-Leffler function defined by

$$
E_{\alpha, \beta}(z)=\sum_{n=0}^{\infty} \frac{z^{n}}{\Gamma(\beta n+\alpha)}
$$

Assume that $\phi_{n}(x)$ is the solution of the following Sturm-Liouville problem:

$$
\left\{\begin{array}{l}
-\phi_{x x}(x)=\lambda \phi(x), \quad 0<x<1, \\
\phi(0)=0, \quad \phi_{x}(1)=0, \quad 0<t<T .
\end{array}\right.
$$

The Neumann-type measured output data at the boundary $x=0$ in terms of $v(x, t)$ can be written in the following form:

$$
k(0)\left(v_{x}(0, t)+\psi_{1}(t)\right)=f(t), \quad t \in(0, T] .
$$

In order to arrange the above solution, let us define the following:

$$
\begin{aligned}
& z_{n}(t)=\left\langle\zeta(\theta), \phi_{n}(\theta)\right| E_{\alpha, 1}\left(-\lambda_{n} t^{\alpha}\right), \\
& w_{n}(t)=\int_{0}^{t} s^{\alpha-1} E_{\alpha, \alpha}\left(-\lambda_{n} s^{\alpha}\right)\left\langle\xi(\theta, t-s), \phi_{n}(\theta)\right\rangle d s .
\end{aligned}
$$

The solution in terms of $z_{n}(t)$ and $w_{n}(t)$ can then be rewritten in the following form:

$$
v(x, t)=\sum_{n=1}^{\infty} z_{n}(t) \phi_{n}(x)+\sum_{n=1}^{\infty} w_{n}(t) \phi_{n}(x) .
$$

Differentiating both sides of the above identity with respect to $x$ and substituting $x=0$ yields

$$
v_{x}(0, t)=\sum_{n=1}^{\infty} z_{n}(t) \phi_{n}^{\prime}(0)+\sum_{n=1}^{\infty} w_{n}(t) \phi_{n}^{\prime}(0) .
$$


Taking into account the over-measured data $k(0)\left(v_{x}(0, t)+\psi_{1}(t)\right)=f(t)$,

$$
f(t)=k(0)\left(\psi_{1}(t)+\sum_{n=1}^{\infty} z_{n}(t) \phi_{n}^{\prime}(0)+\sum_{n=1}^{\infty} w_{n}(t) \phi_{n}^{\prime}(0)\right)
$$

is obtained, which implies that $f(t)$ can be determined analytically. Substituting $t=0$ into this yields

$$
f(0)=k(0)\left(\psi_{1}(0)+\sum_{n=1}^{\infty} z_{n}(0) \phi_{n}^{\prime}(0)\right)
$$

Hence we obtain the following explicit formula for the value $k(0)$ of the unknown coefficient $k(x)$

$$
k(0)=\frac{f(0)}{\psi_{1}(0)+\sum_{n=1}^{\infty} z_{n}(0) \phi_{n}^{\prime}(0)}
$$

Under the determined value $k(0)$, the set of admissible coefficients can be defined as follows:

$$
\mathcal{K}_{0}:=\left\{k(x) \in C^{1}[0,1]: c_{1}>k(x) \geq c_{0}>0, x \in[0,1], k(0)=\frac{f(0)}{\psi_{1}(0)+\sum_{n=1}^{\infty} z_{n}(0) \phi_{n}^{\prime}(0)}\right\} .
$$

The right-hand side of identity (4) defines the input-output mapping $\Phi[k]$ on the set of admissible source functions $\mathcal{K}$

$$
\Phi[k](t):=k(0)\left(\psi_{1}(t)+\sum_{n=1}^{\infty} z_{n}(t) \phi_{n}^{\prime}(0)+\sum_{n=1}^{\infty} w_{n}(t) \phi_{n}^{\prime}(0)\right), \quad \forall t \in[0, T]
$$

The following lemma implies the relation between the parameters $k_{1}(x), k_{2}(x) \in \mathcal{K}_{0}$ at $x=0$ and the corresponding outputs $f_{j}(t):=k(0) u_{x}\left(0, t ; p_{j}\right), j=1,2$.

Lemma 1 Let $v_{1}(x, t)=v\left(x, t ; k_{1}\right)$ and $v_{2}(x, t)=v\left(x, t ; k_{2}\right)$ be the solutions of direct problem (2), corresponding to the admissible parameters $k_{1}(x), k_{2}(x) \in \mathcal{K}_{0}$. Iff $_{j}(t)=u_{x}\left(0, t ; k_{j}\right), j=1,2$, are the corresponding outputs. If the condition $k_{1}(0)=k_{2}(0)=k(0)$, then the outputs $f_{j}(t)$, $j=1,2$, satisfy the following integral identity:

$$
\Delta f(t)=k(0) \sum_{n=1}^{\infty} \Delta w_{n}(t) \phi_{n}^{\prime}(0)
$$

for each $t \in(0, T]$, where $\Delta f(t)=f_{1}(t)-f_{2}(t), \Delta w_{n}(t)=w^{1}(t)-w^{2}(t)$.

Proof By using identity (4), the measured output data $f_{j}(t):=k(0)\left(v_{x}(0, t)+\psi_{1}(t)-\psi_{0}(t)\right)$, $j=1,2$, can be written as follows:

$$
\begin{aligned}
& f_{1}(\tau)=k(0)\left(\psi_{1}(\tau)+\sum_{n=1}^{\infty} z_{n}^{1}(t) \phi_{n}^{\prime}(0)+\sum_{n=1}^{\infty} w_{n}^{1}(t) \phi_{n}^{\prime}(0)\right), \\
& f_{2}(\tau)=k(0)\left(\psi_{1}(\tau)+\sum_{n=1}^{\infty} z_{n}^{2}(t) \phi_{n}^{\prime}(0)+\sum_{n=1}^{\infty} w_{n}^{2}(t) \phi_{n}^{\prime}(0)\right),
\end{aligned}
$$


respectively. Note that the definition of $z_{n}(t)$ implies that $z_{n}^{1}(t)=z_{n}^{2}(t)$. Hence, the difference of these formulas implies the desired result.

The lemma and the definitions of $w_{n}(t)$ and $z_{n}(t)$ given above enable us to reach the following conclusion.

Corollary 1 Let the conditions of Lemma 1 hold. If in addition

$$
\left\langle\xi_{1}(x, t)-\xi_{2}(x, t), \phi_{n}(x)\right\rangle=0, \quad \forall t \in(0, T], \forall n=0,1, \ldots
$$

holds, where

$$
\begin{aligned}
& \xi_{1}(x, t)=\left(\left(k_{1}(x)-1\right) v_{1 x}(x, t)\right)_{x}-x D_{t}^{\alpha} \psi_{1}(t)-D_{t}^{\alpha} \psi_{0}(t)+k_{1}^{\prime}(x) \psi_{1}(t), \\
& \xi_{2}(x, t)=\left(\left(k_{2}(x)-1\right)=v_{2 x}(x, t)\right)_{x}-x D_{t}^{\alpha} \psi_{1}(t)-D_{t}^{\alpha} \psi_{0}(t)+k_{2}^{\prime}(x) \psi_{1}(t),
\end{aligned}
$$

then $f_{1}(t)=f_{2}(t), \forall t \in[0, T]$.

Proof If $\left\langle\xi_{1}(x, t)-\xi_{2}(x, t), \phi_{n}(x)\right\rangle=0, \forall n=0,1, \ldots$, then $k_{1}(x)=k_{2}(x)$. If $k_{1}(x)=k_{2}(x)$, then $u_{1}(x, t)=u_{2}(x, t)$. Since $f(t)$ depends on $u(x, t)$, then from the uniqueness of solution $f_{1}(t)=$ $f_{2}(t)$.

Since $\phi_{n}(x), \forall n=0,1,2, \ldots$ form a basis for the space and $\phi_{n}^{\prime}(0) \neq 0, \forall n=0,1,2, \ldots$, then $k_{1}(x) \neq k_{2}(x)$ implies that $\left\langle\xi_{1}(x, t)-\xi_{2}(x, t), \phi_{n}(x)\right\rangle \neq 0$ at least for some $n \in \mathcal{N}$. Hence by Lemma 1 we conclude that $f_{1}(t) \neq f_{2}(t)$, which leads us to the following consequence: $k_{1}(x) \neq$ $k_{2}(x)$ implies that $\Phi\left[k_{1}\right] \neq \Phi\left[k_{2}\right]$.

Theorem 1 Let conditions (C1), (C2) hold. Assume that $\Phi[\cdot]: \mathcal{K}_{0} \rightarrow C^{1}[0, T]$ is the input-output mapping defined by (4) and corresponding to the measured output $f(t):=$ $k(0) u_{x}(0, t)$. In this case the mapping $\Phi[k]$ has the distinguishability property in the class of admissible parameters $\mathcal{K}_{0}$, i.e.,

$$
\Phi\left[k_{1}\right] \neq \Phi\left[k_{2}\right], \quad \forall k_{1}, k_{2} \in \mathcal{K}_{0} \quad \Rightarrow \quad k_{1}(x) \neq k_{2}(x) .
$$

\section{An analysis of the inverse problem with given measured data $h(t)$}

Consider now the inverse problem with one measured output data $h(t)$ at $x=1$. Taking into account the over-measured data $\left(v(1, t)+\psi_{0}(t)+\psi_{1}(t)\right)=h(t)$,

$$
h(t)=\left(\psi_{0}(t)+\psi_{1}(t)+\sum_{n=1}^{\infty} z_{n}(t) \phi_{n}(1)+\sum_{n=1}^{\infty} w_{n}(t) \phi_{n}(1)\right)
$$

is obtained, which implies that $h(t)$ can be determined analytically.

The set of admissible coefficients can be defined as follows:

$$
\mathcal{K}_{1}:=\left\{k(x) \in C^{1}[0,1]: c_{1}>k(x) \geq c_{0}>0, x \in[0,1]\right\} .
$$

The right-hand side of identity (5) defines the input-output mapping $\Psi[k]$ on the set of admissible parameters $\mathcal{K}$ :

$$
\Psi[k](t):=\left(\psi_{0}(t)+\psi_{1}(t)+\sum_{n=1}^{\infty} z_{n}(t) \phi_{n}(1)+\sum_{n=1}^{\infty} w_{n}(t) \phi_{n}(1)\right), \quad \forall t \in[0, T] .
$$


The following lemma implies the relation between the parameters $k_{1}(x), k_{2}(x) \in \mathcal{K}_{1}$ at $x=1$ and the corresponding outputs $h_{j}(t):=u\left(1, t ; k_{j}\right), j=1,2$.

Lemma 2 Let $v_{1}(x, t)=v\left(x, t ; k_{1}\right)$ and $v_{2}(x, t)=v\left(x, t ; k_{2}\right)$ be the solutions of direct problem (2), corresponding to the admissible parameters $k_{1}(x), k_{2}(x) \in \mathcal{K}_{1}$. If $h_{j}(t)=u\left(1, t ; k_{j}\right), j=1,2$, are the corresponding outputs. The outputs $h_{j}(t), j=1,2$, satisfy the following integral identity:

$$
\Delta h(t)=\sum_{n=1}^{\infty} \Delta w_{n}(t) \phi_{n}(1)
$$

for each $t \in(0, T]$, where $\Delta h(t)=h_{1}(t)-h_{2}(t), \Delta w_{n}(t)=w^{1}(t)-w^{2}(t)$.

Proof By using identity (5), the measured output data $h_{j}(t):=v(1, t)+\psi_{0}(t)+\psi_{1}(t), j=1,2$, can be written as follows:

$$
\begin{aligned}
& h_{1}(t)=\psi_{0}(t)+\psi_{1}(t)+\sum_{n=1}^{\infty} z_{n}^{1}(t) \phi_{n}(1)+\sum_{n=1}^{\infty} w_{n}^{1}(t) \phi_{n}(1), \\
& h_{2}(t)=\psi_{0}(t)+\psi_{1}(t)+\sum_{n=1}^{\infty} z_{n}^{2}(t) \phi_{n}(1)+\sum_{n=1}^{\infty} w_{n}^{2}(t) \phi_{n}(1),
\end{aligned}
$$

respectively. Note that the definition of $z_{n}(t)$ implies that $z_{n}^{1}(t)=z_{n}^{2}(t)$. Hence, the difference of these formulas implies the desired result.

The lemma and the definitions given above enable us to reach the following conclusion.

Corollary 2 Let the conditions of Lemma 2 hold. If, in addition,

$$
\left\langle\xi_{1}(x, t)-\xi_{2}(x, t), \phi_{n}(x)\right\rangle=0, \quad \forall t \in(0, T], \forall n=0,1, \ldots
$$

holds, where

$$
\begin{aligned}
& \xi_{1}(x, t)=\left(\left(k_{1}(x)-1\right) v_{1 x}(x, t)\right)_{x}-x D_{t}^{\alpha} \psi_{1}(t)-D_{t}^{\alpha} \psi_{0}(t)+k_{1}^{\prime}(x) \psi_{1}(t), \\
& \xi_{2}(x, t)=\left(\left(k_{2}(x)-1\right) v_{2 x}(x, t)\right)_{x}-x D_{t}^{\alpha} \psi_{1}(t)-D_{t}^{\alpha} \psi_{0}(t)+k_{2}^{\prime}(x) \psi_{1}(t),
\end{aligned}
$$

then $h_{1}(t)=h_{2}(t), \forall t \in[0, T]$.

Proof If $\left\langle\xi_{1}(x, t)-\xi_{2}(x, t), \phi_{n}(x)\right\rangle=0, \forall n=0,1, \ldots$, then $k_{1}(x)=k_{2}(x)$. If $k_{1}(x)=k_{2}(x)$, then $u_{1}(x, t)=u_{2}(x, t)$. Since $h(t)$ depends on $u(x, t)$, then from the uniqueness of solution $h_{1}(t)=$ $h_{2}(t)$.

Since $\phi_{n}(x), \forall n=0,1,2, \ldots$ form a basis for the space and $\phi_{n}^{\prime}(0) \neq 0, \forall n=0,1,2, \ldots$, then $k_{1}(x) \neq k_{2}(x)$ implies that $\left\langle\xi_{1}(x, t)-\xi_{2}(x, t), \phi_{n}(x)\right\rangle \neq 0$ at least for some $n \in \mathcal{N}$. Hence by Lemma 2 we conclude that $h_{1}(t) \neq h_{2}(t)$, which leads us to the following consequence: $k_{1}(x) \neq k_{2}(x)$ implies that $\Psi\left[k_{1}\right] \neq \Psi\left[k_{2}\right]$.

Theorem 2 Let conditions (C1), (C2) hold. Assume that $\Psi[\cdot]: \mathcal{K}_{1} \rightarrow C[0, T]$ is the inputoutput mapping defined by (6) and corresponding to the measured output $h(t):=u(1, t)$. 
In this case the mapping $\Psi[k]$ has the distinguishability property in the class of admissible parameters $\mathcal{K}_{1}$, i.e.,

$$
\Psi\left[k_{1}\right] \neq \Psi\left[k_{2}\right], \quad \forall k_{1}, k_{2} \in \mathcal{K}_{1} \quad \Rightarrow \quad k_{1}(x) \neq k_{2}(x) .
$$

\section{Conclusion}

The aim of this study was to investigate the distinguishability properties of the inputoutput mappings $\Phi[\cdot]: \mathcal{K} \rightarrow C^{1}[0, T]$ and $\Psi[\cdot]: \mathcal{K} \rightarrow C[0, T]$, which are determined by the measured output data at $x=0$ and $x=1$, respectively. In this study, we conclude that the distinguishability of the input-output mappings $\Phi[\cdot]$ and $\Psi[\cdot]$ holds, which implies the injectivity of the inverse mappings $\Phi^{-1}$ and $\Psi^{-1}$. This provides the insight that compared to the Dirichlet type, the Neumann-type measured output data is more effective for the inverse problems of determining unknown coefficients. Moreover, the measured output data $f(t)$ and $h(t)$ are obtained analytically by a series representation, which leads to the explicit form of the input-output mappings $\Phi[\cdot]$ and $\Psi[\cdot]$. We also show that the value of the unknown coefficient $k(x)$ at $x=0$ is determined by using the Neumann-type measured output data at $x=0$, which brings more restrictions on the set of admissible coefficients. However, $k(1)$ is not obtained by the Dirichlet-type measured output data at $x=1$. This provides the insight that the Neumann-type measured output data is more effective than that of Dirichlet type for the inverse problems of determining an unknown coefficient. This work advances our understanding of the use of the Fourier method of separation of variables and the input-output mapping in the investigation of inverse problems for fractional parabolic equations. The author plans to consider various fractional inverse problems in future studies, since the method discussed has a wide range of applications.

Competing interests

The authors declare that they have no competing interests.

Authors' contributions

All authors contributed equally and significantly in writing this paper. All authors read and approved the final manuscript.

Author details

'Department of Mathematics, Faculty of Science and Literature, Izmir University of Economics, Sakarya Caddesi, No. 156, Balcova, Izmir, 35330, Turkey. ${ }^{2}$ Department of Mathematics, Kocaeli University, Umuttepe, Izmit, Kocaeli 41380, Turkey.

\section{Acknowledgements}

The research was supported in part by the Scientific and Technical Research Council (TUBITAK) of Turkey and Izmir University of Economics.

Received: 6 November 2013 Accepted: 14 May 2014 Published: 27 May 2014

References

1. Canon, J, Lin, Y: An inverse problem of finding a parameter in a semi-linear heat equation. J. Math. Anal. Appl. 145, 470-484 (1990)

2. Canon, J, Lin, Y: Determination of a parameter $p(t)$ in some quasi-linear parabolic differential equations. Inverse Probl. 4, 35-44 (1998)

3. Canon, J, Lin, Y: Determination of source parameter in parabolic equations. Meccanica 27, 85-94 (1992)

4. Dehgan, M: Identification of a time-dependent coefficient in a partial differential equation subject to an extra measurement. Numer. Methods Partial Differ. Equ. 21, 621-622 (2004)

5. Demir, A, Ozbilge, E: Analysis of a semigroup approach in the inverse problem of identifying an unknown coefficient. Math. Methods Appl. Sci. 31, 1635-1645 (2008)

6. Demir, A, Ozbilge, E: Semigroup approach for identification of the unknown coefficient in a quasi-linear parabolic equation. Math. Methods Appl. Sci. 30, 1283-1294 (2007)

7. Demir, A, Hasanov, A: Identification of the unknown diffusion coefficient in a linear parabolic equation by the semigroup approach. J. Math. Anal. Appl. 340, 5-15 (2008)

8. Fatullayev, A: Numerical procedure for the simultaneous determination of unknown coefficients in a parabolic equation. Appl. Math. Comput. 164, 697-705 (2005) 
9. Isakov, V: Inverse Problems for Partial Differential Equations. Springer, New York (1998)

10. Renardy, M, Rogers, RC: An Introduction to Partial Differential Equations. Springer, New York (2004)

11. Showalter, RE: Monotone Operators in Banach Spaces and Nonlinear Partial Differential Equations. Am. Math. Soc., Providence (1997)

12. Luchko, Y: Initial boundary value problems for the one dimensional time-fractional diffusion equation. Fract. Calc. Appl. Anal. 15, 141-160 (2012)

10.1186/1687-2770-2014-134

Cite this article as: Ozbilge and Demir: Analysis of the inverse problem in a time fractional parabolic equation with mixed boundary conditions. Boundary Value Problems 2014, 2014:134

Submit your manuscript to a SpringerOpen ${ }^{\circ}$ journal and benefit from:

- Convenient online submission

- Rigorous peer review

- Immediate publication on acceptance

- Open access: articles freely available online

- High visibility within the field

- Retaining the copyright to your article 